\title{
SÍNTESE E CARACTERIZAÇÃO DE NITROSIL COMPLEXOS COM AÇÃO ANTIBACTERIANA FRENTE A Pseudomonas aeruginosa
}

\author{
Thuanny M. de Sousa ${ }^{a}$, Dayana P. S. Penha ${ }^{a}$, Daniel de L. Pontes ${ }^{a}$, Ana C. F. de B. Pontes ${ }^{a}$ e Francisco O. N. da Silva ${ }^{\text {a*, }}$ \\ anstituto de Química, Universidade Federal do Rio Grande do Norte, 59078-970, Natal - RN, Brasil
}

Recebido em 29/08/2019; aceito em 27/11/2019; publicado na web em 10/02/2020

\begin{abstract}
SYNTHESIS AND CHARACTERIZATION OF NITROSYL COMPLEXES WITH ANTIBACTERIAL ACTION AGAINST Pseudomonas aeruginosa. The compounds nitrosyl cis-[Ru(bpy)(phen)TU(NO) $]\left(\mathrm{PF}_{6}\right)_{3}$ (compound 1) and cis-[Ru(bpy) $($ phen $)(4-\mathrm{N}-\mathrm{Imd})(\mathrm{NO})]\left(\mathrm{PF}_{6}\right)_{3}$ (compound 2) $\left(\mathrm{bpy}=2,2^{\prime}\right.$-bipyridine, phen $=1,10$ ' -phenanthroline, $\mathrm{TU}=$ thiourea and 4-N-Imd = 4-nitroimidazole) were synthesized and characterized by UV-visible, infrared spectroscopies and electrochemical techniques. In the study of the reactivity it was possible to verify the nitric oxide labilization by square wave voltammetry and a photochemical upon blue light irradiation. This feature is very important for a potential application in phototherapy. Additionally, the antibacterial activity of the nitrosyl complexes was tested against gram-negative bacteria Pseudomonas aeruginosa. Thus, it has been observed that the complexes are capable of inhibiting the growth of such microorganisms.
\end{abstract}

Keywords: ruthenium; nitrix oxide; Pseudomonas aeruginosa.

\section{INTRODUÇÃO}

A molécula de óxido nítrico tornou-se uma das mais importantes moléculas de sinalização intercelular identificada nos anos $1980 .{ }^{1-3}$ A descoberta de que o NO está envolvido em múltiplos processos fisiológicos como a neurotransmissão, ${ }^{4-7}$ pressão arterial ${ }^{8-11}$ e participação na capacidade do sistema imunológico no combate a células tumorais e parasitas intracelulares ${ }^{12-16}$ tem expandido a quantidade de pesquisas no desenvolvimento de novos compostos capazes de liberar essa molécula.

Sínteses e estudos sobre a cinética desses compostos têm sido de fundamental importância para viabilizar sua aplicação como metalofármacos. ${ }^{17}$ Nitrosilo complexos de rutênio têm despertado bastante interesse devido à boa estabilidade térmica, baixa toxicidade, solubilidade em água e capacidade de liberar NO de modo fotoquímico e através de redução química. ${ }^{18-20}$

A formação de complexos de rutênio doadores de NO em alvosbiológicos, também dependem da interação bioquímica dos ligantes coordenados ao centro metálico. Os ligantes polipiridínicos, tais como 2,2'-bipiridina e 1-10'-fenantrolina apresentam coordenação bidentada e por serem quelatos resultam em compostos de coordenação relativamente estáveis, ${ }^{21,22}$ proporcionando uma intercalação na interação com o $\mathrm{DNA}^{23}$ de modo a apresentar ação como agente antitumoral ${ }^{24-26}$ e agente antimicrobiano. ${ }^{27}$

Atualmente, uma grande preocupação alegada pela Organização Mundial da Saúde (OMS) são as mortes causadas por doenças bacterianas. ${ }^{28} \mathrm{O}$ uso de antibióticos como alternativa para solucionar esse problema tem se tornado ineficaz, devido à grande resistência adquirida por esses microorganismos ${ }^{29,30} \mathrm{e}$ ao uso incorreto e indiscriminado desses medicamentos, os quais conduz a difíceis tratamentos..$^{31-37}$

Uma estratégia utilizada para favorecer a atividade antimicrobiana é a complexação de moléculas a centros metálicos, ${ }^{38,39}$ sendo esse método uma alternativa viável no controle de infecções, pois compostos de coordenação com ligantes biologicamente relevantes são normalmente mais ativos do que os ligantes livres. ${ }^{40,41}$

Visando o desenvolvimento de novos compostos com potencial atividade biológica frente a bactérias, apresentamos a caracterização,

\footnotetext{
*e-mail: ordeleisilva@yahoo.com.br
}

o comportamento fotoquímico e a atividade antimicrobiana dos compostos cis-[Ru(bpy)(phen)L( $\left.\left.\mathrm{NO}^{+}\right)\right]\left(\mathrm{PF}_{6}\right)_{3}$, com L = 4-nitroimidazol (4-N-Imd) e tiouréia (TU) contra Pseudomonas aeruginosa.

\section{EXPERIMENTAL}

\section{Reagentes}

$\mathrm{O}$ tricloreto de rutênio hidratado - $\mathrm{RuCl}_{3} \cdot \mathrm{XH}_{2} \mathrm{O}$ (Sigma Aldrich; $99 \%$ ) foi utilizado como material de partida para as sínteses dos complexos. Os ligantes 2,2'-bipiridina, tiouréia, 4-nitroimidazol e 1,10'-fenantrolina foram adquiridos da Sigma-Aldrich e utilizados sem qualquer purificação adicional. cis-[Ru(bpy)(phen) $\left.\mathrm{Cl}_{2}\right]$ foi preparado de acordo com procedimento descrito na literatura. ${ }^{42}$

\section{Sínteses dos compostos}

\section{Síntese dos complexos cis-[Ru(bpy)(phen $\left.)\left(\mathrm{NO}_{2}\right)\right]\left(\mathrm{PF}_{6}\right)$}

Esse composto foi preparado pela dissolução de $0,100 \mathrm{~g}$ $(0,190 \mathrm{mmol})$ do complexo cis-[Ru(bpy)(phen) $\left.\mathrm{Cl}_{2}\right]$ com $0,026 \mathrm{~g}$ $(0,228 \mathrm{mmol})$ para o ligante 4-nitroimidazol (4-N-Imd) e 0,019 g $(0,228 \mathrm{mmol})$ para a tiouréia (TU) em uma mistura de $10 \mathrm{~mL}$ etanol e água (1:1) sob condições de refluxo. Após 2 h adicionou-se nitrito de sódio $(0,228 \mathrm{mmol})$ deixando nas mesmas condições para produzir os nitro complexos cis-[Ru(bpy)(phen) $\left.\mathrm{L}\left(\mathrm{NO}_{2}\right)\right]\left(\mathrm{PF}_{6}\right)$. A mistura foi concentrada num evaporador rotativo até um volume de $8 \mathrm{~mL}$, e o sólido foi precipitado pela adição de $2 \mathrm{~mL}$ de $\mathrm{NH}_{4} \mathrm{PF}_{6}$ $0,89 \mathrm{~mol} \mathrm{~L}^{-1} \mathrm{O}$ sólido foi separado por filtração e armazenado sob vácuo. Rendimento: $72 \%$ ( $\mathrm{L}=4$-nitroimidazol) e $51 \%(\mathrm{~L}=$ tiouréia). $\mathrm{C}_{25} \mathrm{H}_{21} \mathrm{~F}_{6} \mathrm{~N}_{8} \mathrm{O}_{5} \mathrm{PRu}(759,07)$ : calcd. C 39,52\%, H 2,76\%, N 14,75\%; obtido: C 39,22\%, H 2,62\%, N 14,49\%. $\mathrm{C}_{23} \mathrm{H}_{22} \mathrm{~F}_{6} \mathrm{~N}_{7} \mathrm{O}_{3} \mathrm{PRuS}(722,07)$ : calcd. C 38,22\%, H 3,04\%, N 13,57\%, S 4,43\%; obtido: C 39,02\%, H 2,82\%, N 14,09\%, S 4,08\%. Esses dados foram obtidos em um analisador elementar CHN 2400 da Perkin-Elmer.

\section{Síntese do complexo cis-[Ru(bpy)(phen $) L(N O)]\left(P F_{6}\right)_{3}$}

Os complexos $c i s-[\mathrm{Ru}(\mathrm{bpy})(\mathrm{phen}) \mathrm{L}(\mathrm{NO})]\left(\mathrm{PF}_{6}\right)_{3}$ foram preparados pela dissolução de $0,100 \mathrm{~g}(0,144 \mathrm{mmol})$ cis-[Ru(bpy)(phen) $\left.\mathrm{L}\left(\mathrm{NO}_{2}\right)\right]$ $\left(\mathrm{PF}_{6}\right)$ em $5 \mathrm{~mL}$ metanol sob uma atmosfera de argônio. A essa mistura 
foi adicionada uma solução concentrada de ácido trifluoroacético ( $2 \mathrm{~mL}$ ) para promover a conversão de $\mathrm{NO}_{2}^{-}$em $\mathrm{NO}^{+}$. Após $2 \mathrm{~h}$, a mistura foi concentrada num evaporador rotativo até um volume de $7 \mathrm{~mL}$, e o sólido foi precipitado pela adição de $2 \mathrm{~mL}$ de $\mathrm{NH}_{4} \mathrm{PF}_{6}$ $0,89 \mathrm{~mol} \mathrm{~L}^{-1}$. O sólido foi separado por filtração e armazenado sob vácuo. Rendimento: 69\% (Para o composto cis-[Ru(bpy)(phen) (4-N-Imd)NO] $\left.\left(\mathrm{PF}_{6}\right)_{3}\right)$ e $59 \%$ (Para o composto cis-[Ru(bpy)(phen) (TU)NO] $\left.\left(\mathrm{PF}_{6}\right)_{3}\right) . \mathrm{C}_{25} \mathrm{H}_{21} \mathrm{~F}_{18} \mathrm{~N}_{8} \mathrm{O}_{4} \mathrm{P}_{3} \mathrm{Ru}(1033,07)$ : calcd. C $29,04 \%$, H 2,03\%, N 10,84\%; obtido: C 29,22\%, H 2,14\%, N 10,79\%. $\mathrm{C}_{23} \mathrm{H}_{22} \mathrm{~F}_{18} \mathrm{~N}_{7} \mathrm{O}_{2} \mathrm{P}_{3} \mathrm{RuS}(996,07)$ : calcd. C 27,70\%, H 2,20\%, N 9,84\%, S 3,21\%; obtido: C 28,02\%, H 2,34\%, N 9,96\%, S 3,19\%.

\section{Equipamentos}

Os espectros de absorção eletrônica foram registrados em um espectrofotômetro Uv-visível Agilent, modelo 8453, usando células de quartzo com $1 \mathrm{~cm}$ de caminho ótico. Os espectros de absorção no infravermelho foram obtidos em pastilhas de $\mathrm{KBr}$, em um espectrofotômetro da Shimadzu, modelo FTIR-8400S, série IRAffinity-1, software IRSOLUTION, versão 1.60, com número de varredura de 32 e resolução $4 \mathrm{~cm}^{-1}$. A análise de RMN 1D e 2D foram adquiridos em espectrômetro BRUKER Avance $300 \mathrm{MHz}$, em dimetilsulfóxido deuterado (DMSO- $\mathrm{d}_{6}$ ). Nos espectros de ${ }^{1} \mathrm{H}$, os deslocamentos químicos foram referenciados por picos de hidrogênio pertencentes ao resíduo não-deuterado em relação ao TMS. As medidas eletroquímicas foram realizadas em um potenciostato modelo Epsilon da Bioanalytical Systems, Inc (BASi). Foi utilizada uma célula eletroquímica constituída por três eletrodos: $\mathrm{Ag} / \mathrm{AgCl}$ como eletrodo de referência, eletrodo de trabalho de carbono vítreo e o auxiliar de platina. O eletrólito utilizado foi NaTFA $0,1 \mathrm{~mol} \mathrm{~L}^{-1}$ e $\mathrm{pH}=3,0$. As soluções analisadas foram desaeradas borbulhando argônio de alta pureza.

\section{Estudo computacional}

Os cálculos de otimização das estruturas dos complexos foram obtidos utilizando o programa Software Gaussian 09 (Gaussian Inc., Wallingford, CT) através da Teoria Funcional de Densidade (DTF) com o funcional híbrido B3LYP (restrito). ${ }^{43-45}$ Nos cálculos, foi utilizado o modelo de solvatação contínua polarizada para simular o efeito do solvente. ${ }^{46} \mathrm{O}$ conjunto de bases do potencial efetivo relativístico LANL2DZ ${ }^{47,48}$ foi usado para o átomo de Ru e o conjunto de bases 6-31G (d) para os outros átomos. ${ }^{47,48} \mathrm{~A}$ Teoria Funcional de Densidade Dependente do tempo (TD-DFT) ${ }^{48}$ foi utilizada para calcular os espectros de UV-Visível a partir das estruturas otimizadas por DFT. Para a obtenção dos valores de energia e contribuições percentuais dos orbitais envolvidos nas transições eletrônicas bem como a simulação do efeito do solvente, foram utilizados os softwares Multiwfn e GaussSum 3.0. ${ }^{49}$

\section{Ensaios bacterianos}

\section{Microrganismo e condições de cultura}

Foi utilizada neste trabalho a seguinte cepa de referência da American Type Culture Collection (ATCC): bactéria Gram-negativa Pseudomonas aeruginosa ATCC 9027. A avaliação da atividade antimicrobiana iniciou-se com o pré-inóculo, a qual foi preparado repicando a cepa do estoque em placas de Agar BHI, em seguida deixou-se na estufa por 24 horas a temperatura de $37^{\circ} \mathrm{C}$. Após esse período, preparou-se o inóculo ressuspendendo colônias do pré-inóculo em solução salina (soro fisiológico 0,9\%) até atingir turbidez de 0,5 na escala McFarland, correspondente a $10^{8} \mathrm{UFC} / \mathrm{mL}$ (unidade formadora de colônia por mililitro). Após o período de incubação, a cultura bacteriana foi ajustada para a concentração final de $10^{6}$ unidades formadoras de colônias $\mathrm{UFC} / \mathrm{mL}$.

\section{Concentração inibitória mínima (CIM)}

A triagem da atividade antimicrobiana foi obtida utilizando o método de microdiluição em caldo, partindo da metodologia descrita por $C L S I,{ }^{50}$ com algumas modificações. De acordo com essa metodologia, foram adicionados $100 \mu \mathrm{L}$ do caldo Mueller Hinton $(\mathrm{MH})$ em cada um dos poços, juntamente com adição de $100 \mu \mathrm{L}$ do composto avaliado no primeiro poço, e em seguida transferindo-se $100 \mu \mathrm{L}$ dessa mistura caldo-composto para os demais poços, realizando as diluições seriadas. Posteriormente, adicionou-se $100 \mu \mathrm{L}$ de inóculo bacteriano a mistura caldo-composto contida em cada um dos poços, e em seguida as placas foram incubadas durante 18 horas a temperatura de $35 \pm 2{ }^{\circ} \mathrm{C}$ sob agitação $(200 \mathrm{rpm})$ e a avaliação da densidade óptica foi realizada em um leito de microplacas (Epoch Biotek, Winooski, EUA) no comprimento de $595 \mathrm{~nm}$. Os compostos foram diluídos em Agar BHI (Brain Heart Infusion) contendo 4\% de dimetilsulfóxido (DMSO) na concentração inicial de $800 \mu \mathrm{mol} \mathrm{L}-1$ para o complexo 1 e $400 \mu \mathrm{mol} \mathrm{L}^{-1}$ para o complexo 2. Os resultados da atividade antibacteriana dos compostos testados foram reportados pelas curvas de crescimento bacteriano versus concentração, obtidas pelo programa GraphPad Prism 5, e todas as curvas foram normalizadas de 0 a $100 \%$. O crescimento bacteriano foi determinado em porcentagem a partir da razão da densidade ótica (DO) obtida em 595 $\mathrm{nm}$ no ensaio antibacteriano pela do controle positivo.

\section{RESULTADOS E DISCUSSÃO}

\section{Caracterização dos complexos}

Os espectros eletrônicos dos nitro complexos cis-[Ru(bpy)(phen) $\left.\mathrm{L}\left(\mathrm{NO}_{2}\right)\right]^{+}(\mathrm{L}=4-\mathrm{N}-\mathrm{Imd}, \mathrm{TU})$ obtidos em meio aquoso apresentaram bandas intraligantes do tipo $\pi \rightarrow p *$ em 264 e $286 \mathrm{~nm}$ referente aos ligantes fenantrolina e bipiridina, respectivamente. ${ }^{51,52}$ Tais transições são consistentes àquelas observadas para $\left[\mathrm{Ru}(\mathrm{phen})_{2}(\mathrm{ppn})\right]^{2+}$ $\left[\mathrm{Ru}(\mathrm{bpy})_{2}(\mathrm{ppn})\right]^{2+}$ em $262 \mathrm{~nm}$ e $285 \mathrm{~nm}$, respectivamente. ${ }^{53}$ Além disso, as transições de transferência de carga de metal para ligando (MLCT) são observadas na faixa de 405 a $420 \mathrm{~nm}$.

Em comparação aos nitro complexos, o nitrosil mostrou um deslocamento para o azul das bandas MLCT $\mathrm{d} \pi\left(\mathrm{Ru}^{\mathrm{II}}\right) \rightarrow \pi^{*}$ (bpy/phen) (de 420 para $330 \mathrm{~nm}$ ) devido à grande estabilização dos orbitais $\mathrm{d} \pi$ do metal, causada pela forte interação back-bonding do ligante $\mathrm{NO}^{+}{ }^{54} \mathrm{O}$ espectro ainda apresenta uma transição de baixa absortividade molar na região de $476 \mathrm{~nm}$ atribuída a MLCT $\mathrm{d} \pi\left(\mathrm{Ru}^{\mathrm{II}}\right) \rightarrow \pi^{*}\left(\mathrm{NO}^{+}\right)$. Essa transição é comumente observada na faixa de $420-480 \mathrm{~nm}$ para uma série de nitrosil complexos com tetraminas $\left[\mathrm{Ru}\left(\mathrm{NH}_{3}\right)_{4}(\mathrm{NO}) \mathrm{X}\right]^{\mathrm{n}+}$, em que $\mathrm{X}=\mathrm{OH}^{-}, \mathrm{Cl}^{-}, \mathrm{Br}^{-}$e I-, $\mathrm{n}=2 ; \mathrm{X}=\mathrm{OH}_{2}, \mathrm{n}=3 .{ }^{55,56}$

Cálculos TD-DFT são relatados para o complexo 2, em que o espectro calculado apresenta uma ótima concordância com o experimental quando o ligante NO encontra-se trans ao ligante bipiridina (Figura 1). Assim, os dados do TD-DFT foram utilizados para reforçar a atribuição das transições eletrônicas.

A banda em $267 \mathrm{~nm}$ ocorre com maior contribuição dos orbitais HOMO-2 $\rightarrow$ LUMO+6 (51\%) atribuída à transição intraligante da fenantrolina. A banda em 294 tem maior contribuição dos orbitais HOMO-1 e LUMO+4 (33\%) referente à intraligante da bipiridina. Por último, a banda em $448 \mathrm{~nm}$ apresenta contribuição dos orbitais HOMO-4 $\rightarrow$ LUMO (29\%) correspondente à transição MLCT dos orbitais $\mathrm{d} \pi$ do rutênio para os orbitais $\pi^{*}$ do NO. O espectro eletrônico experimental do complexo 2 e seu espectro simulado apresentaram concordância ao analisar as energias das bandas com as contribuições dos orbitais (Figura 1). Além disso, as porcentagens relativas das 
(A)

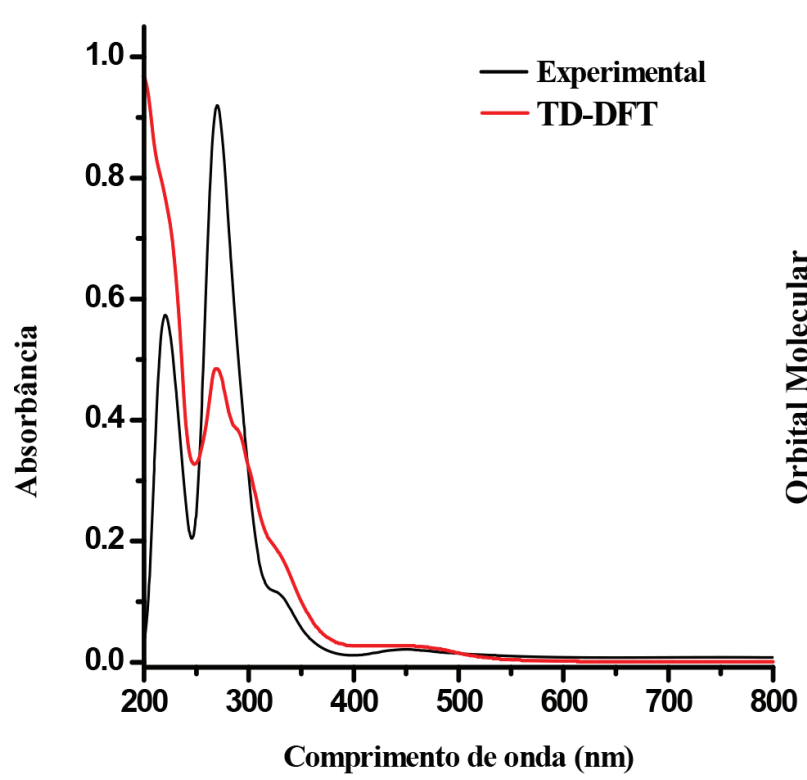

(B)

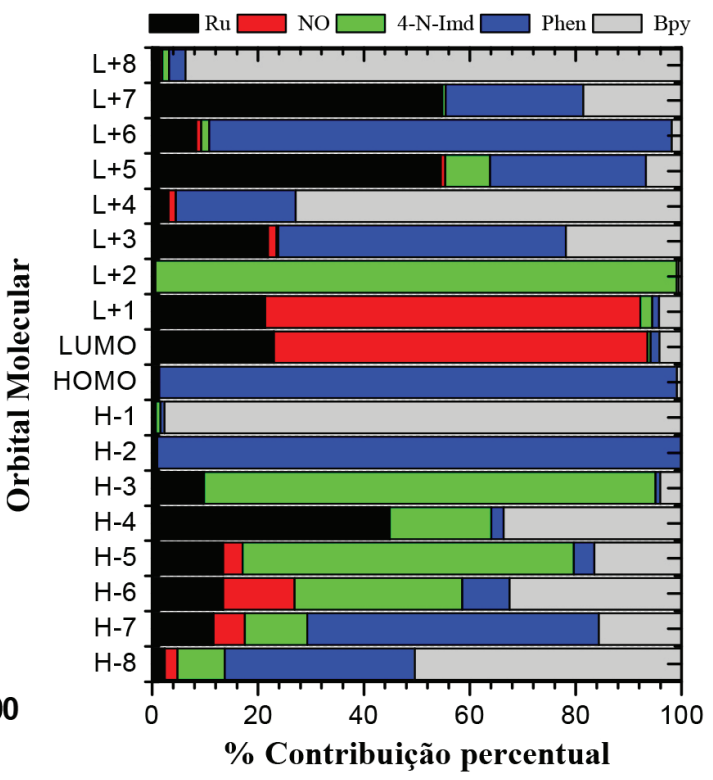

Figura 1. Espectro UV-Visível do complexo 2 em solução aquosa (A) Experimental (-) e calculado (-). (B). Percentual de contribuição dos orbitais moleculares selecionados

contribuições atômicas dos orbitais HOMO e LUMO são observadas na Figura $1 \mathrm{~b}$ e indicam maior semelhança entre os dados quando o NO está trans a bipiridina.

No acompanhamento ácido-base com os nitrosil complexos (Figura 1S) observou-se que os espectros eletrônicos apresentaram uma dependência com relação ao $\mathrm{pH}$. Dessa forma, à medida que aumenta o pH das soluções contendo os complexos, verificam-se bandas intensas na faixa 400-420 nm que são atribuídas à transição MLCT $\mathrm{d} \pi\left(\mathrm{Ru}^{\mathrm{II}}\right) \rightarrow \pi^{*}$ (bpy/phen) dos nitro complexos formados conforme o equilíbrio exibido na equação $1 .^{54,57-59}$

$$
\begin{aligned}
& \text { cis- }[\mathrm{Ru}(\text { bpy })(\text { phen }) \mathrm{L}(\mathrm{NO})]^{+\mathrm{n}}+2 \mathrm{OH}-\rightleftharpoons \\
& \text { cis- }\left[\mathrm{RU}(\text { bpy })(\text { phen }) \mathrm{L}\left(\mathrm{NO}_{2}\right)\right]^{+\mathrm{n}}+\mathrm{H}_{2} \mathrm{O}
\end{aligned}
$$

No gráfico (Figura 2) de pH vs. absorbância verifica-se uma inflexão indicando um equilíbrio referente a interconversão $\mathrm{NO}^{+}$para $\mathrm{NO}_{2}{ }^{-}$em $\mathrm{pH}$ de 5,9 para o complexo com ligante 4-nitroimidazol e 6,7 para o complexo com a tiouréia. A diferença dos ligantes da esfera de coordenação influencia diretamente na intensidade da retrodoação que ocorre do metal para o ligante nitrosil, de maneira a alterar seu caráter eletrofílico. Assim, a constante de equilíbrio aumenta quanto maior a capacidade receptora dos ligantes coordenados ao metal.

Ao relacionar, na Tabela 1, os valores da constante de equilíbrio, frequência de estiramento e potencial de meia onda do par redox $\mathrm{NO}^{+/ 0}$ dos nitrosil complexos presentes nesse trabalho e na literatura, ${ }^{43}$ verifica-se que ao aumentar a deficiência eletrônica sobre o grupo nitrosil há uma intensificação no caráter eletrofílico deste ligante. ${ }^{58}$ Por isso, constata-se uma maior frequência no estiramento do $\mathrm{NO}^{+}$ e um maior potencial de redução. ${ }^{60}$

Os espectros de infravermelho para os nitrosil complexos apresentam como principal característica o estiramento NO na região entre 1926 a $1942 \mathrm{~cm}^{-1}$ (Figura 2S). Esses valores associados ao movimento vibracional deste ligante indicam que o mesmo encontra-se coordenado ao centro metálico na forma $\mathrm{NO}^{+}{ }^{58,61-66} \mathrm{De}$ acordo com a literatura, quando o NO está coordenado pela geometria linear observa-se na região do infravermelho elevadas frequências de estiramento, como consequência do menor comprimento da ligação M-N e susceptibilidade de sofrer ataques nucleofílicos. ${ }^{67,68}$

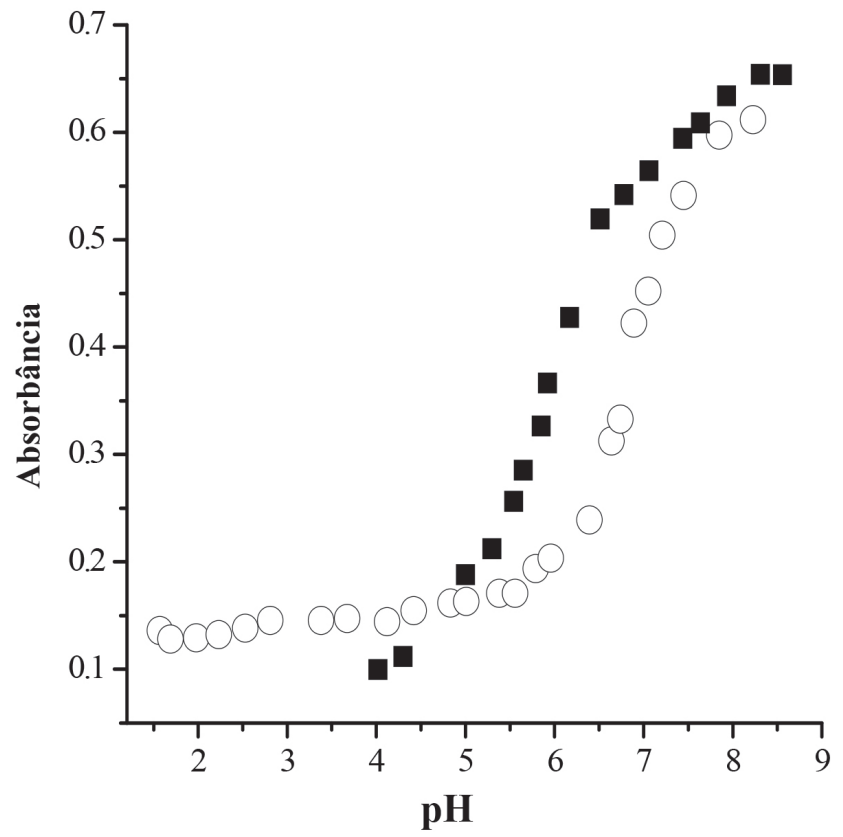

Figura 2. Titulação espectrofotométrica dos complexos cis-[Ru(bpy)(phen) $(\mathrm{TU}) \mathrm{NO}]\left(\mathrm{PF}_{6}\right)_{3}(\mathrm{O})$ e cis-[Ru(bpy)(phen)(4-N-Imd)NO](PF$)_{3}(\mathbf{\square})$, nos $l_{\max }$ de 422 e $417 \mathrm{~nm}$, respectivamente

Outras bandas que se destacam nos espectros vibracionais são atribuídas ao vN-H do ligante tiouréia em 3411 e $\delta\left(\mathrm{NH}_{2}\right)$ em $1607 \mathrm{~cm}^{-1}$ no complexo 1 e as frequências em 3426, 1523, 1381 e $1321 \mathrm{~cm}^{-1}$ são típicas do ligante 4-nitroimidazol no complexo 2. Além dessas, verificam-se bandas em comum aos complexos associadas aos ligantes polipiridínicos, em torno de 1523-1451, 1433-1384, 716-770 e 845-558 $\mathrm{cm}^{-1}$ atribuídas aos $v \mathrm{C}=\mathrm{C}, \nu \mathrm{C}=\mathrm{N}, \delta \mathrm{C}-\mathrm{H}_{\text {e } ~} \mathrm{PF}_{6}^{-}$, respectivamente..$^{43}$

Para uma melhor caracterização dos nitrosil complexos, os espectros de RMN, 2D COSY e HSQC foram obtidos (Figuras 3S, $4 \mathrm{~S}$ e $5 \mathrm{~S}$ ). A Figura 3 ilustra os espectros de hidrogênio para ambos os compostos. A presença de todos os sinais do anel N-heterocíclico 
Tabela 1. Valores da $\mathrm{K}_{\text {eq }}, \mathrm{NO}^{+}$e $\mathrm{E}_{1 / 2} \mathrm{NO}^{+/ 0}$ referentes aos complexos cis-[Ru(bpy)(phen)LNO $]^{+n}$

\begin{tabular}{|c|c|c|c|}
\hline Complexo & $\mathrm{K}_{\mathrm{eq}}$ & $\mathrm{vNO}^{+}\left(\mathrm{cm}^{-1}\right)$ & $\mathrm{E}_{1 / 2}(\mathrm{mV})\left(\mathrm{NO}^{+/ 0}\right)$ \\
\hline$c i s-[\mathrm{Ru}(\mathrm{bpy})(\mathrm{phen})(\mathrm{TU}) \mathrm{NO}]\left(\mathrm{PF}_{6}\right)_{3}$ & $3,06 \times 10^{13}$ & 1926 & 32 \\
\hline cis-[Ru(bpy)(phen)(4-N-Imd)NO]($\left(\mathrm{PF}_{6}\right)_{3}$ & $6,10 \times 10^{18}$ & 1942 & 117 \\
\hline$c i s-\left[\mathrm{Ru}(\mathrm{bpy})_{2}(\mathrm{TU}) \mathrm{NO}\right]\left(\mathrm{PF}_{6}\right)_{3}{ }^{54}$ & $1,42 \times 10^{15}$ & 1932 & 37,5 \\
\hline cis-[Ru(bpy $\left.)_{2}(\operatorname{ImN}) \mathrm{NO}\right]\left(\mathrm{PF}_{6}\right)^{56}$ & $1,74 \times 10^{16}$ & 1944 & 200 \\
\hline
\end{tabular}

(7,1 e 7,3 ppm) sugere que o ligante 4-nitroimidazol está coordenado ao centro de metal através do átomo de nitrogênio (Tabela 1S). Com base no aparecimento de 16 sinais para os átomos hidrogênio, os espectros de RMN 2D dos compostos e comportamento similar descrito para sistemas de rutênio com ligantes polipiridínicos ${ }^{56,69}$ sugerem que todos átomos de hidrogênio não são equivalentes magneticamente.

O voltamograma cíclico dos nitrosil complexos constata-se um único processo quase reversível referente ao par redox $\mathrm{NO}^{+/ 0}$, cujo potencial de meia-onda está em $32 \mathrm{mV}$ para o complexo 1 e $117 \mathrm{mV}$ para o complexo 2. A Figura 4 apresenta o voltamograma cíclico do complexo 2 em $\mathrm{NaCF}_{3} \mathrm{COO} 0,1 \mathrm{~mol} \mathrm{~L}^{-1}$. Ao comparar os valores de potenciais de meia-onda, observa-se que os complexos apresentam valores distintos em virtude do tipo predominante de interação (doador sigma ou receptor $\pi$ ) exercida pelos ligantes auxiliares L. Assim, o caráter $\pi$ receptor do ligante 4-nitroimidazol no complexo 2 provoca no centro metálico uma deficiência de elétrons que enfraquece a transferência de carga do rutênio para o grupo nitrosil. Deste modo o NO experimenta uma diminuição na densidade eletrônica, a qual dificulta o processo redox. Quando o ligante coordenado é a tiouréia no complexo 1 , observa-se um valor de potencial redox mais baixo devido ao maior poder $\sigma$ doador deste ligante, fazendo que haja um aumento de densidade eletrônica sobre o NO. Vale ressaltar que na janela de potencial analisada não é possível observar o potencial referente ao par redox $\mathrm{Ru}^{3+/ 2+}$, pois ao coordenar o grupo nitrosil o valor de potencial associado ao centro metálico é observado acima de $2,0 \mathrm{~V}^{68,70}$
Com o objetivo de reforçar esta atribuição, os voltamogramas de onda quadrada para os complexos cis- $\left[\mathrm{Ru}(\mathrm{bpy})\left(\text { phen }(\mathrm{L})\left(\mathrm{NO}^{+}\right)\right]^{\mathrm{n+}}\right.$ foram obtidos por uma mudança no tempo de polarização do eletrodo de trabalho, a -0,10 para ambos os compostos (Figuras 6S e 7S, respectivamente). Para esses complexos, observou-se um processo com maior intensidade de corrente em $32 \mathrm{mV}$ para o complexo 1 e em $117 \mathrm{mV}$ para o complexo 2, referente à oxidação que ocorre no ligante nitrosil de $\mathrm{NO}^{0}$ para $\mathrm{NO}^{+}$e ainda, um segundo processo de menor corrente em $592 \mathrm{mV}$ e $745 \mathrm{mV}$, respectivamente, atribuído a oxidação do centro metálico de rutênio no aqua complexo formado. Isso ocorre porque o processo de redução $\mathrm{NO}^{+} \rightarrow \mathrm{NO}^{0}$ é seguido por uma reação química, como pode-se observar nas equações propostas 2,3 e 4, , $^{43}$ em que ocorre a labilização do $\mathrm{NO}^{0}$ e coordenação de uma molécula de água.

$$
\begin{aligned}
& \text { cis- }[\mathrm{Ru}(\text { bpy })(\text { phen }) \mathrm{L}(\mathrm{NO})]^{+\mathrm{n}}+\mathrm{e}^{-} \rightleftharpoons \\
& \text { cis- }\left[\mathrm{Ru}(\text { bpy })(\text { phen }) \mathrm{L}\left(\mathrm{NO}^{0}\right)\right]^{+\mathrm{n}} \\
& \text { cis- }\left[\mathrm{Ru}^{2+}(\mathrm{bpy})(\mathrm{phen}) \mathrm{L}\left(\mathrm{NO}^{0}\right)\right]^{+\mathrm{n}}+\mathrm{H}_{2} \mathrm{O} \rightleftharpoons \\
& \text { cis- }\left[\mathrm{Ru}^{2+}(\mathrm{bpy})(\text { phen }) \mathrm{L}\left(\mathrm{H}_{2} \mathrm{O}\right)\right]^{+\mathrm{n}}+\mathrm{NO}^{0} \\
& \text { cis- }\left[\mathrm{Ru}^{2+}(\mathrm{bpy})(\text { phen }) \mathrm{L}\left(\mathrm{H}_{2} \mathrm{O}\right)\right]^{+\mathrm{n}} \rightleftharpoons \\
& \text { cis- }\left[\mathrm{Ru}^{3+}(\mathrm{bpy})(\text { phen }) \mathrm{L}\left(\mathrm{H}_{2} \mathrm{O}\right)\right]^{+\mathrm{n}}+\mathrm{e}^{-}
\end{aligned}
$$

Com o aumento do tempo de polarização, constatou-se um aumento da corrente de pico relacionada ao processo redox $\mathrm{Ru}^{\mathrm{III} / \mathrm{II}}$. Um comportamento semelhante é encontrado para outros complexos semelhantes, conforme relatado na literatura. ${ }^{56,71}$

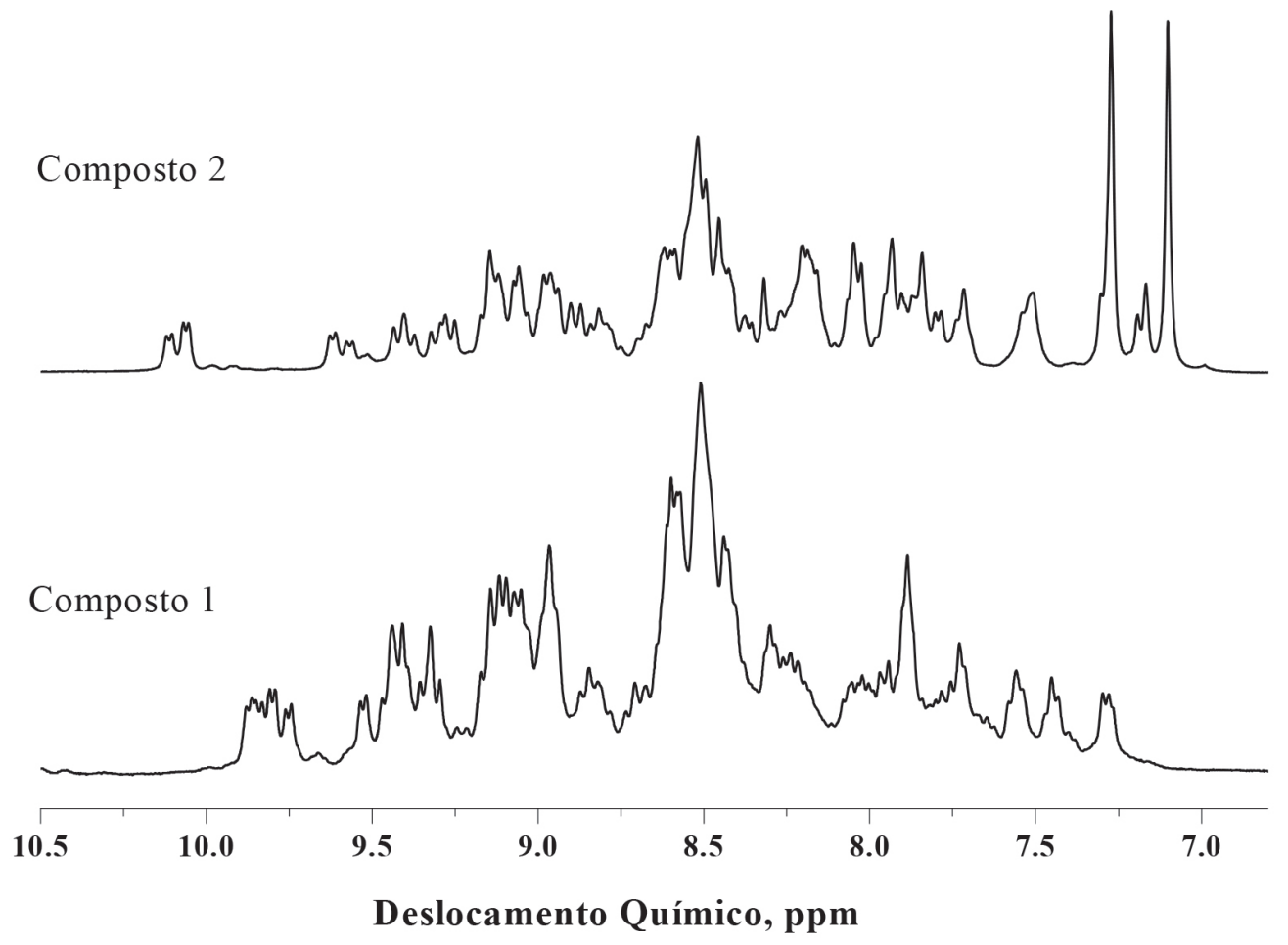

Figura 3. Espectros $R M N$ de ${ }^{l} H$ para os compostos 1 e 2, em DMSO-d 


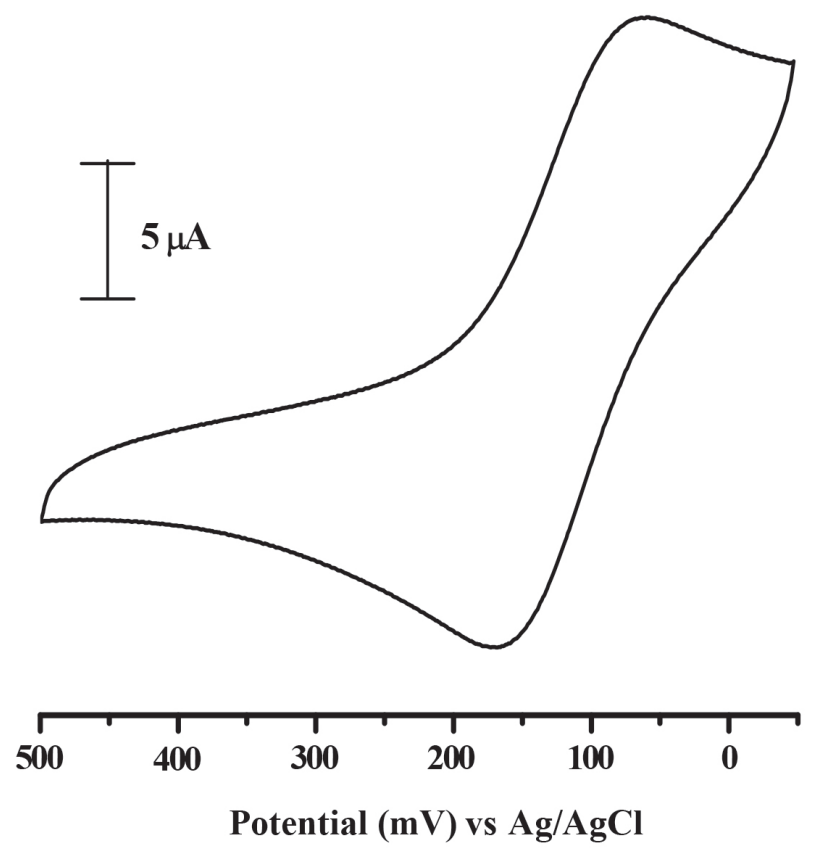

Figura 4. Voltamograma cíclico a $100 \mathrm{mV} / \mathrm{s}$ para o eletrodo de carbono vítreo na presença do ion complexo $\left[\mathrm{Ru}(\text { bpy)(phen) }(4-\mathrm{N}-\mathrm{Imd}) \mathrm{NO}]^{3+}\right.$ em solução aquosa de $\mathrm{NaCF}_{3} \mathrm{COO} 0,1 \mathrm{~mol} \mathrm{~L}^{-1} \mathrm{pH} \mathrm{3,0}$

\section{Estudo fotoquímico}

A irradiação com LED azul nos complexos 1 e 2 foi realizada para determinar qualitativamente a liberação do óxido nítrico da esfera de coordenação. As fotólises foram acompanhadas por espectroscopia eletrônica em diferentes tempos de exposição ao LED. Ao analisar os espectros eletrônicos dos complexos, verifica-se que ao aumentar o tempo de exposição ocorre o aumento da intensidade da transição de transferência de carga na região entre 400 e 500 nm (Figura 8S). Dessa forma, as modificações no espectro sugerem que ocorreu liberação do ligante nitrosil. A irradiação de nitrosil complexos de rutênio em solução aquosa com fótons de energia resulta na redução do óxido nítrico e posteriormente, sua liberação, de modo que em seu lugar coordena-se uma molécula de água, formando o aqua complexo. ${ }^{43,72,73}$

$\mathrm{Na}$ literatura Rose e Mascharak ${ }^{74}$ propuseram dois tipos de mecanismos para liberação de $\mathrm{NO}$ de forma fotoquímica. O primeiro produz as espécies $\mathrm{NO}$ e $\left[\mathrm{Ru}^{3+}\right.$-solvente], enquanto o segundo $\mathrm{NO}$ e $\left[\mathrm{Ru}^{2+}\right.$-solvente], como produtos finais, equação 5 .

$$
\begin{aligned}
c i s-\left[\mathrm{Ru}^{2+}(\text { bpy })(\text { phen }) \mathrm{L}\left(\mathrm{NO}^{+}\right)\right]^{\mathrm{n+}} \stackrel{\mathrm{hv}}{\longrightarrow} & c i s-\left[\mathrm{Ru}^{3+}(\text { bpy })(\mathrm{phen}) \mathrm{L}\left(\mathrm{NO}^{0}\right)\right]^{\mathrm{n+}}+\mathrm{H}_{2} \mathrm{O}+\mathrm{e}^{-} \\
& c i s-\left[\mathrm{Ru}^{2+}(\text { bpy })(\text { phen }) \mathrm{L}\left(\mathrm{H}_{2} \mathrm{O}\right)\right]^{\mathrm{n+}}+\mathrm{NO}^{0}
\end{aligned}
$$

É constatado que o primeiro mecanismo ocorre quando os nitrosil complexos de rutênio apresentam ligantes como amina, carboxamida, tiolato, heme e fenolato, já no segundo os complexos têm na esfera de coordenação ligantes polipiridínicos e base de Schiff. Nesse mecanismo o retorno da espécie $\mathrm{Ru}^{2+}$, após a fotólise, parece proceder de uma redução espontânea da espécie transitória $\mathrm{Ru}^{3+}$. Analisando os complexos 1 e 2, as irradiações apresentaram perfil compatível com o produto final do segundo mecanismo, devido às semelhanças do metal, estado de oxidação e ligantes..$^{54,74}$

Com o intuito de ajudar nesta atribuição, foi realizado o acompanhamento eletroquímico desta reação (Figura 5). Nota-se que no primeiro ciclo voltamograma não há nenhuma espécie eletroativa na janela de potencial aplicado. Após 1 hora de irradiação com o LED azul, percebe-se o aparecimento de um processo eletroquímico em
0,590 V atribuído ao par redox $\mathrm{Ru}^{\mathrm{IIIII}}$ do aqua complexo formado após a redução e labilização do óxido nítrico. Com base nesses resultados, constata-se um comportamento similar entre os dois compostos estudados neste trabalho com sistemas relatados na literatura. ${ }^{70,71}$

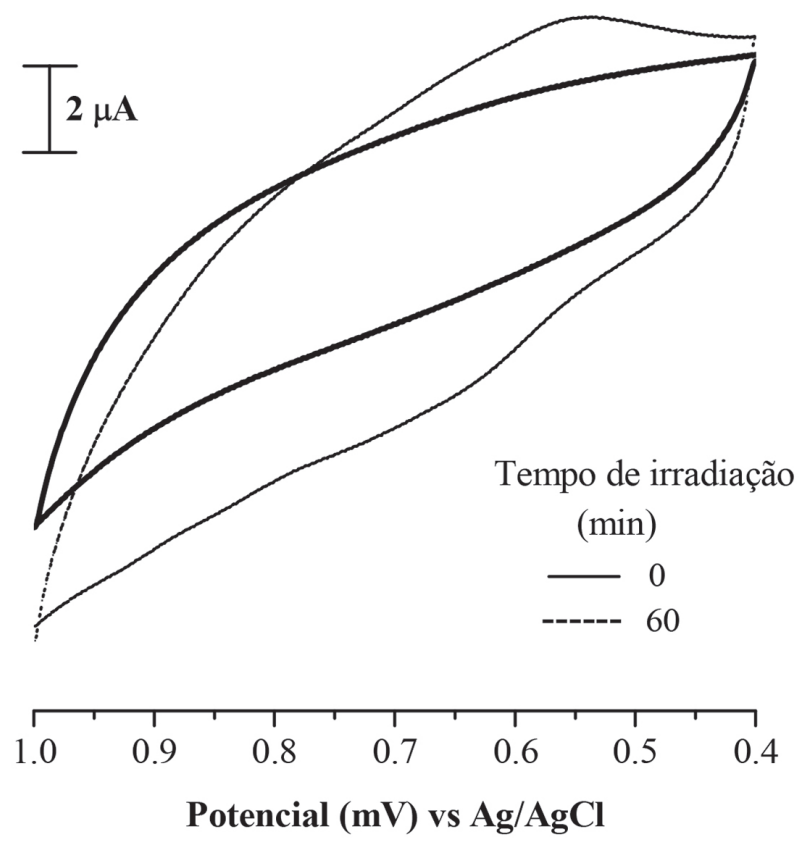

Figura 5. Voltamogramas cíclicos a $100 \mathrm{mV} \mathrm{s}^{-1}$ do eletrodo de carbono vítreo em solução de NaTFA 0,1 mol L $1(p H=3,5)$ contendo o composto 2 antes e depois da irradiação sob LED azul

\section{Atividade antibacteriana frente a Pseudomonas aeruginosa}

A atividade antibacteriana do nitrosilo complexo foi testada frente à bactéria de cepa gram-negativa Pseudomonas aeruginosa ATCC - 9027 (Figura 6). Desse modo, foi observado que o complexo 1 (Concentração Inibitória Mínima $\left.(\mathrm{CIM})=121,5 \mathrm{mmol} \mathrm{L}^{-1}\right)$ é capaz de inibir o crescimento das bactérias Pseudomonas aeruginosa (ATCC - 9027) numa concentração bem menor que o complexo 2 $\left(\mathrm{CIM}>500 \mathrm{mmol} \mathrm{L}^{-1}\right)$. Segundo a literatura, existem alguns fatores que são importantes no que diz respeito aos complexos metálicos apresentarem atividade antimicrobiana, dentre esses, estão o efeito quelato e a carga total dos complexos. Em relação ao efeito quelato, verifica-se que complexos formados por ligantes que se coordenam aos centros metálicos de forma bidentada, como a 1,10'-fenantrolina, a 2,2'-bipiridina e a 2,2'-dipiridilamina, apresentam maior eficiência antimicrobiana quando comparado a complexos que possuem ligantes monodentados, como a piridina. Já no caso da carga total dos complexos constata-se que a atividade antimicrobiana decresce na seguinte ordem: catiônico $>$ neutro $>$ aniônico. ${ }^{75}$

Adicionalmente, relatos da literatura demonstra que os ligantes bipiridina, fenantrolina, 4-nitroimidazol e tiouréia livres de coordenação não possuem atividade antibacteriana. Dessa forma, a capacidade de inibir o crescimento da Pseudomonas aeruginosa é decorrente de características intrínsecas do complexo formado, não sendo atribuída isoladamente a cada um dos ligantes. A concentração inibitória mínima (CIM) reportada para as hidrazonas e seus complexos de cobre (II) contra Pseudomonas aeruginosa apresentaram valores que variam de $232 \mu \mathrm{mol} \mathrm{L}-1$ a valores maiores que $570 \mu \mathrm{mol} \mathrm{L}{ }^{-1}{ }^{76}$ Além disso, elevados valores da CIM obtidos, na faixa entre 1532 a $1357 \mu \mathrm{mol} \mathrm{L}^{-1}$, referentes à coordenação do $\mathrm{Cu}$ (II) a derivados de tiossemicarbazonas, sulfatiazol e nimesulida, ${ }^{77}$ e do composto $\left[\mathrm{Ru}(\right.$ mctz)(bpy)(dppf) $] \mathrm{PF}_{6}$ descritos na literatura $\left(\mathrm{CIM}>500 \mathrm{mmol} \mathrm{L}^{-1}\right),{ }^{78}$ não foram considerados 


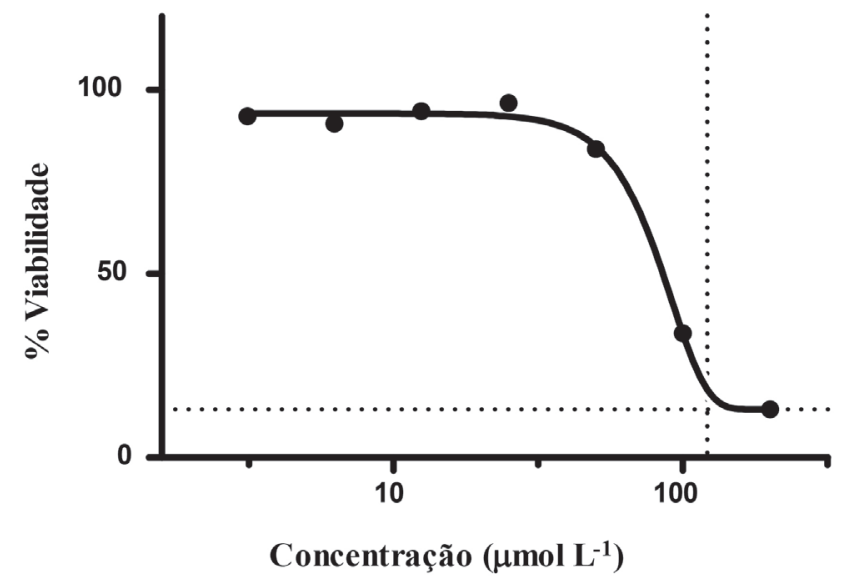

Figura 6. Porcentagem de viabilidade do inóculo pela concentração do complexo 1 frente as bactérias Pseudomonas aeruginosa

como uma boa alternativa frente a essa bactéria Gram-negativa. ${ }^{79} \mathrm{Com}$ isso, pode-se observar que o composto em estudo apresenta menor concentração para inibir o crescimento de bactérias gram-negativas, indicando que o mesmo pode ser considerado um bacteriostático.

\section{CONCLUSÃO}

Os novos nitrosil complexos de rutênio foram obtidos com eficiência de acordo com as técnicas analisadas, sendo capazes de liberar NO por irradiação com luz azul e redução eletroquímica. Ao analisar os ensaios antibacterianos frente às bactérias Pseudomonas aeruginosa, observou-se que composto 1 apresentou maior capacidade de inibir o crescimento dessa bactéria Gram-negativa em comparação a sistemas relatados na literatura, ${ }^{75-79}$ podendo ser considerado como bacteriostático.

\section{MATERIAL SUPLEMENTAR}

Estão disponíveis em http://quimicanova.sbq.org.br, na forma de arquivo PDF, com acesso livre, espectros eletrônicos de acompanhamento da interconversão $\mathrm{NO}^{+} / \mathrm{NO}_{2}{ }^{-}$dos compostos (Figura 1S), espectros vibracionais na região do infravermelho para os nitrosil complexos (Figura 2S), dados referentes aos deslocamentos químicos dos hidrogênios dos ligantes fenantrolina e bipiridina (Tabela $1 \mathrm{~S})$, espectros de ressonância magnética nuclear 2D dos compostos (Figuras 3S e 4S), representação estrutural dos compostos (Figura $5 \mathrm{~S}$ ), voltamogramas de onda quadrada (Figuras $6 \mathrm{~S}$ e $7 \mathrm{~S}$ ) e espectros eletrônico mostrando a mudança do perfil em função da exposição à luz azul (Figura 8S).

\section{AGRADECIMENTOS}

Os autores agradecem ao Conselho Nacional de Desenvolvimento Científico e Tecnológico (CNPq) e à Coordenação de Aperfeiçoamento de Pessoal de Nível Superior (CAPES) pelo apoio financeiro. À Centro Nacional de Processamento de Alto Desempenho - Universidade Federal do Ceará (CENAPAD), em particular a Tércio de F. Paulo, pela contribuição nos cálculos teóricos. Ao laboratório de Microbiologia Clínica em nome de Maísa Rocha Abrantes da UFRN pela realização dos ensaios antibacterianos.

\section{REFERÊNCIAS}

1. Ignarro, L. J. B.; Nitric Oxide: Biology and Phatology, Academic Press: Cambridge, 2000.
2. Sauaia, M. G.; Lima, R. G.; Tedesco, A. C.; Silva, R. S.; J. Am. Chem. Soc. 2003, 125, 14718.

3. Yetik-Anacak, G.; Catravas, J. D.; Vasc. Pharm. 2006, 45, 268.

4. Sotirov, E.; Dobreva, G.; Noeva, A.; Papasov, M.; General Pharmacology: The Vacular System. 1996, 27, 395.

5. Moncada, S.; Higgs, E. A.; Br. J. Pharmacol. 2006, 147,193.

6. Vincent, S. R.; Prog. in Neurob. 2010, 90, 246.

7. Zullino, S.; Buzzella, F.; SimoncinI, T.; Vasc. Pharm. 2018, 110, 71.

8. Thomas, S. R.; Witting, P. K.; Drummond, G. R.; Antioxid. Redox Signaling 2008, 10, 1713.

9. Costa, E. D.; Silva, J. F.; Aires, R. D.; Garcia, D. C.; Kansaon, M .J.; Wainstein, A. J.; Rezende, B. A.; Teixeira, M. M.; Silva, R. F.; Cortes, S. F.; Lemos, V. S.; Nitric Oxide. 2018, 80, 98.

10. Mel, A.; Murad, F; Seifalian, A. M.; Chem. Rev. 2011, 111, 5742.

11. Socco, S.; Bovee, R. C.; Palczewski, M. B.; Hickok, J. R.; Thomas, D. D.; Pharm. Res. 2017, 121, 52.

12. Silva, J. J. N.; Osakabe, A. L.; Pavanelli, W. R.; Silva, J. S.; Franco, D.; Br. J. Pharmacol. 2007, 152, 112.

13. Sülz, L.; Astorga, G.; Bellete, B.; Iturriaga, R.; Mackay-sim, A.; Bacigalupo, J.; Nitric Oxide 2009, 20, 238.

14. Gomes, A. J.; Espreafico, E. M.; Tfouni, E.; Mol. Pharmaceutics 2013, $10,544$.

15. Wang, Y.; Chen, M.; Hong, H.; Wang, Y.; Li, Q.; Liu, H.; Yang, M.; Hong, F.; Yang, S.; Arc. Med. Res. 2018, 49, 219.

16. Lok, H. C.; Sahni, S.; Richardson, V.; Kalinowski, D. S.; Kovacevic, Z.; Lane, D. J. R.; Richardson, D. R.; Free Rad. Biol. Med. 2014, 75, 14.

17. Figueiredo, L. E.; Cilli, E. M.; Molina, R. A. S.; Espreafico, E. M.; Tfouni, E.; Inor. Chem. Commun. 2013, 28, 60.

18. Tfouni, E.; Truzzi, D. R.; Tavares, A.; Gomes, A. J.; Figueiredo, L. E.; Franco, D, W.; Nitric Oxide 2012, 26, 38.

19. Caramori, G. F.; Ortolan, A. O.; Parreira, R. L. T.; Silva, E. H.; J. Org. Chem. 2015, 54, 799.

20. Roose, M.; Sasaki, I.; Bukhanko, V.; Mallet-Ladeira, S.; Barba-Barba, R. M.; Ortiz, G. R; Enriquez-Cabrera, A.; Farfán, N.; Lacroix, P. G.; Malfant, I.; Polyhedron 2018, 151, 100.

21. Moreno, Y.; Spodine, A.; Vega, A.; Saillard, J.; Inor. Chim. Acta 2003, 350,651 .

22. Bencini, A.; Lippolis, V.; Chem. Rev. 2010, 254, 2096.

23. Donnici, C. L.; Oliveira, I. M. F.; Temba, E. S. C.; Castro, M. C. R.; Quim. Nova 2002, 25, 668.

24. Krishnamurti, C.; Saryan, L. A.; Petering D. H.; Cancer Res. 1980, 40, 4092.

25. Smith, A, P.; Frases, L.; Comp. Coord. Chem. II 2005, 1,1.

26. Kellett, A.; Howe, O.; O'connor, M.; Mccann, M.; Creaven, B. S.; Mcclean, S.; Kia, A. F.; Case, A.; Devereux, M.; Free Rad. Biol. Med. 2012, 53, 564.

27. Mengjun, C.; Jianding, C.; Mohamed, T.; Chin. J. Chem. 2012, 30, 1531.

28. Barbosa, G, A.; Silva, J.; Appelt, P.; Fuganti, O.; Murakami, F. S.; Araujo, M. P.; Inorg. Chem. Commun. 2018, 90, 108.

29. Luo, H.; Xia, Y.; Sun, B.; Huang, L.; Wang, X.; Lou, H.; Zhu, X.; Weidong P., W.; Zhang, X.; Biochem. Res. Intern. 2017, 1.

30. Bolla, P. K.; Kalhapure, R. S.; Rodriguez, V. A.; Ramos, D. V.; Dahl, A.; Renukuntla, J.; J. Drug Delivery Sci. Technol. 2019, 49, 6.

31. Alekshun, M. N.; Levy, S. B.; Elsevier Inc. 2007, 1037.

32. Zhou, Y.; Liu, M.; Rugaie, A.; Osaman, R.; Lekong, X.; Alsam, S.; Battah, S.; Yuanxie, Y.; Hilder, R. C.; Zhou, T.; Eur. J. Med. Chem. 2015, $55,146$.

33. Guimarães, D. O.; Momesso, L. S.; Pupo, M. T.; Quim. Nova 2010, 33, 667.

34. Rocha, D. P.; Pinto, G. F.; Rugiero, R.; Oliveira, C. A.; Guerra, W.; Fontes, A. P. S.; Tavares, T. T.; Marzano, I. M.; Maia, C. P.; Quim. Nova 2011, 34, 111.

35. Ventola, C. L.; Pharmacy and Therapeutics 2015, 40, 277. 
36. Meyer, E.; Gastmeier, P.; Deja, M.; Schwab, F.; Int. J. Med. Microbiol. 2013, 303, 388.

37. Kumar, M.; Sodhi, K. K.; Singh, P.; Agrawal, P. K.; Singh, D. K.; Environmental Nanotechnology, Monitoring and Management 2019, 11,1 .

38. Kalhapure, R. S.; Sonawane, S. J.; Sikwal, D. R.; Jadhav, M.; Rambharose, S.; Mocktar, C.; Govender, T.; Colloid Surf., B 2015, 136, 651.

39. Santi, E.; Facchin, G.; Faccio, R.; Barroso, R. P.; Costa-filho, A. J.; Borthagaray, G.; Torre, M. H.; J. Inorg. Biochem. 2016, 155, 67.

40. Grabchev, I.; Yordanova, S.; Bosch, P.; Vasileva-tonkova, E.; Kukeva, R.; Stoyanov, S.; Stoyanova, R.; J. Mol. Struct. 2017, 1130, 974.

41. Apohan, E.; Yilmaz, U.; Yilmaz, O.; Serindag, A.; Küçükbay, H.; J. Organomet. Chem. 2017, 828, 52.

42. Penha, D. P. S.; Dissertação de Mestrado, Universidade Federal do Rio Grande do Norte, Brasil, 2018.

43. Becke, A. D.; Chem. Phys. 1993, 98, 5648.

44. Lee, C. T.; Yang, W. T.; Parr, R. G.; Phys. Rev. B 1988, 37, 785.

45. Casida, M. E.; Jamorski, C.; Casida, K. C.; Salahub, D. R.; J. Chem. Phys. 1998, 108, 4439

46. Mennucci, B.; Cance, E.; Tomasi, J.; J. Phys. Chem. B 1997, 101, 10506.

47. Wadt, W. R.; Hay, P. J.; J. Chem. Phys. 1985, 82, 299.

48. Jensen, F.; Introduction to Computational Chemistry, $2^{\text {nd }}$ ed., John Wiley: Hoboken, 2007.

49. O’Boyle, N. M.; Tenderholt, A. L.; Langner, K. M.; J. Comput. Chem. 2008, 29, 839.

50. Cockerill, F. R.; Wikler, M. A.; Alder, J.; Dudley, M. N.; Eliopoulos, G. M.; Ferraro, M. J.; Hardy, D. J.; Hecht, D. W.; Hindler, J. A.; Patel, J. B.; Powell, M.; Swenson, J. M.; Thomson, Jr., R. B.; Traczewski, M. M.; Turnidge, J. D.; Weinstein, M. P.; Zimmer, B. L.; Methods for Dilution Antimicrobial Susceptibility Tests for Bacteria That Grow Aerobically, Approved Standard, $9^{\text {a }}$ ed., 2012, p. 32.

51. Callahan, R. W.; Meyer, T. J.; Inorg. Chem. 1977, 16, 574.

52. Boonyavonga, N.; Suwanruji, P.; Hannongbua, S.; Li, F.; Sunc, L.; Karpkirda, T. M.; J. Photochem. Photobiol., A 2014, 287, 40.

53. Li, J.; Sum, Y.; Zhu, Z.; Zhao, H.; Tan, L.; J. Inorg. Biochem. 2016, 161, 128.

54. Cândido, M. C. L.; Oliveira, A. M.; Silva, F. O. N.; Holanda, A. K. M.; Pereira, W. G.; Sousa, E. H. S.; Carneiro, Z. A.; Silva, R. S.; Lopes, L. G. F.; J. Braz. Chem. Soc. 2015, 26, 1824.

55. Pell, S.; Armor, J. N.; Inorg. Chem. 1973, 12, 873.

56. Silva, F. O. N.; Araújo, S. X. B.; Holanda, A. K. M.; Meyer, E.; Sales, F. A. M.; Diógenes, I. C. N.; Carvalho, I. M. M.; Moreira, I. S.; Lopes, L. G. F.; Eur. J. Inorg. Chem. 2006, 2020.

57. Thompson, M. S.; Meyer, T. J.; J. Am. Chem. Soc. 1981, 103, 5577.

58. Tfouni, E.; Krieger, M.; McGarvey, B. R.; Franco, D. W.; Coord. Chem. Rev. 2003, 236, 57
59. Holanda, A. K. M.; Silva, F. O. N.; Carvalho, I. M. M.; Batista, A. A.; Ellena, J.; Castellano, E. E.; Moreira, I. S.; Lopes, L. G. F.; Polyhedron 2007, 26, 4653

60. Bottomley, F.; Acc. Chem. Res. 1978, 11, 158.

61. Rodrigues, F. P.; Carneiro, Z. A.; Mascharak, P.; Curti, C.; Silva, R. S.; Coord. Chem. Rev. 2016, 306, 701.

62. Gomes, M. G.; Davanzo, C. U.; Silva, S. C.; Lopes, L. G. F.; Santos, P. S., Franco, D. W.; J. Chem. Soc. Dalton Trans. 1998, 601.

63. Borges, S. S. S.; Davanzo, C. U.; Castellano, E. E.; Schpector, Z.; Silva, S. C.; Franco, D. W.; Inorg. Chem. 1998, 37, 2670.

64. Lopes, L. G. F.; Wieraszko, A.; El-Sherif, Y.; Clarke, M. J.; Inorg. Chim. Acta. 2001, 312, 15.

65. Wieraszko, A.; Clarke, M. J.; Lang, D. R.; Lopes, L. G. F.; Franco, D. W.; Life Sci. 2001, 68, 1535.

66. Poelhsitz, G. V.; Bogado, A. L.; Souza, G. D.; Rodrigues-Filho, E.; Batista, A. A.; Araujo, M. P.; Inor. Chem. Commun. 2007, 10, 133.

67. Ford, P. C.; Bourassa, J.; Miranda, K.; Lee, B.; Lorkovic, I.; Boggs, S.; Kudo, S.; Laverman, L.; Coord. Chem. Rev. 1998, 171, 185.

68. Kumar, A. Pandey, R.; Gupta, R. K.; Ghosh, K.; Pandey, D. S.; Polyhedron 2013, 52, 837.

69. Silva, C. D. S.; Pazc, I. A.; Abreu, F. D.; de Sousa, A. P.; Veríssimo, C. P.; Nilberto R.F. Nascimento, N. R. F.; Paulo, T. F.; Zampieri, D.; Eberline, M. N.; Gondim, A. C. S.; Andrade, L. C.; Carvalho, I. M. M.; Sousa, E. H. S.; Lopes, L. G. F.; J. Inorg. Biochem, 2018, 83.

70. Silva, F. O. N.; Penha, D. P. S.; Alencar, A. E. V.; Pontes, D. L.; Brito, A. C. F.; Sousa, E. H. S.; Lopes, L. G. F.; Quim. Nova 2018, 41, 400.

71. Sousa, A. P.; Fernandes, A. F.; Paz, I. A.; Nascimento, N. R. F.; Ellena, J.; Sousa, E. H. S.; Lopes, L. G. F.; Holanda, A. K. M.; J. Braz. Chem. Soc. 2017, 28, 2117.

72. Carlos, R. M.; Ferro, A. A.; Silva, H. A. S.; Gomes, M. G.; Borges, S. S. S.; Ford, P. C.; Tfouni, E.; Franco, D. W.; Inor. Chim. Acta 2004, 357, 1381.

73. Lima, R. G.; Sauaia, M. G.; Bonaventura, D.; Tedesco, A. C.; Lopez, R. F. V.; Bendhack, L. M.; Silva, R. S.; Inor. Chim. Acta 2005, 358, 2643.

74. Rose, M. J.; Mascharak, P. K.; Coord. Chem. Rev. 2008, 252, 2093.

75. Efthimiadou, E. K.; Katsaros, N.; Karaliota, A.; Psomas, G.; Inor. Chim. Acta. 2007, 360, 4093.

76. Despaigne, A. A. R.; da Costa, F. B.; Piro, O. E.; Castellano, E. E.; Louro, S. R. W.; Beraldo, H.; Polyhedron 2012, 285.

77. Nunes, J. H. B.; de Paiva, R. E. F.; Cuin, A.; Ferreira, A. M. da C.; Lustri, W. R.; Corbi, P. P.; J. of Mol. Struct. 2016, 1112, 14.

78. Appelt, P.; Silva, J. P.; Fuganti, O.; Aquino, L. E. N.; Sandrino, B.; Wohnrath, K.; Santos, V. A. Q.; Cunha, M. A. A.; Veiga, A.; Murakami, F. S.; Back, D. F.; Araujo, M. P.; J. of Organom. Chem. 2017, 846, 326.

79. Ferraz, K. S. O. Dissertação de Mestrado. Universidade Federal de Minas Gerais, Brasil, 2008. 